\title{
ESTUDIO INICIAL DE GEO-POLÍMEROS A PARTIR DE ARCILLAS
}

Jose Ordoñez ${ }^{1 *}$, Raúl Fajardo²

*A quien debe dirigirse la correspondencia

\begin{abstract}
RESUMEN
El presente artículo describe un estudio inicial de la generación de geo-polímeros asociando comparativamente la reacción geopolimérica del caolín referida por varios autores y una arcilla cerámica local. Los geo-polímeros materiales aglomerantes que en un futuro podrían sustituir a materiales tradicionales de construcción como: cemento, bloque entre otros con un bajo impacto ambiental en su producción y uso, además de tener mayor resistencia mecánica y química.

Los análisis de fluorescencia y difractometría de RX del caolín y de la arcilla cerámica permitió conocer las similitudes tanto químicas como mineralogías de las materias primas, se partió del caolín con una relación $\mathrm{SiO}$ / $\mathrm{Al} 2 \mathrm{O} 3$ de 1.2 para verificar las concentraciones químicas del activador generando una matriz experimental con los iones de $\mathrm{Na}+1, \mathrm{~K}+1, \mathrm{Al}+3$, Si-2 en un medio alcalino. La reactividad entre los iones alcalinos y el ion silicato demostraron iniciar la reacción de geo-polimerización tanto en el caolín como en la arcilla cerámica considerando que la relación $\mathrm{SiO} 2 / \mathrm{Al} 2 \mathrm{O} 3$ de la arcilla es de 1.78. Las briquetas de prueba se desarrollaron con ambas materias primas que fueron tratadas con solución la activadora desarrollada en la primera parte del estudio. Los ensayos dieron como resultados briquetas resistentes al agua con 5 horas de curado y una resistencia de hasta 32 megas pascales en los ensayos de compresión.
\end{abstract}

Palabras clave: Geo-polimerización, activación alcalina, cemento alternativo.

\section{INITIAL STUDY OF GEOPOLYMERS MADE FROM CLAYS}

\begin{abstract}
This article describes an initial study of the generation of geo-polymers associating comparatively reaction geopolimérica kaolin reported by several authors and a local ceramic clay. The geo-polymer binder material in the future could replace traditional building materials such as cement, block and others with low environmental impact in their production and use, in addition to greater mechanical and chemical resistance.

The fluorescence analysis and diffractometry RX kaolin and ceramic clay yielded information both chemical similarities and mineralogy of raw materials, broke kaolin with a $\mathrm{SiO} 2 / \mathrm{Al} 2 \mathrm{O} 3$ ratio of 1.2 to verify chemical concentrations activator generating a matrix with experimental ions $\mathrm{Na}+1, \mathrm{~K}+1, \mathrm{Al}+3, \mathrm{Si}-2$ in an alkaline medium. The reactivity between the alkali ions and silicate ion showed start geo-polymerization reaction both kaolin clay and pottery whereas the $\mathrm{SiO} 2$ / $\mathrm{Al} 2 \mathrm{O} 3$ ratio is 1.78 clay. Briquettes test is developed with both raw materials that were treated with the activating solution developed in the first part of the study. The tests gave results as briquettes waterproof 5 hours of curing and resistance up to 32 megabytes in pascals compression tests.
\end{abstract}

Keywords: Geo polymerization, alkali activation, alternative cement.

1. Universidad de Cuenca, Ecuador, Agustín Cueva y Avenida Doce de Abril. E-mail: Jose.or@icloud.com

2. Universidad de Cuenca, Ecuador, Agustín Cueva y Avenida Doce de Abril. 


\section{INTRODUCCIÓN}

En el último siglo el cemento portland puzolanico se ha convertido en el principal material para el sector de la construcción, por su gran resistencia, fácil uso y su precio accesible. El problema de este tipo de materiales es que para su producción se requiere de la quema de combustibles fósiles generando agentes contaminantes principalmente gases de invernadero como $\mathrm{CO}_{2}$ (Duda, 1977) y sus escombros son un problema ambiental grave.

Para la producción de una tonelada de cemento es necesario emplear $1761000 \mathrm{Kcal}$ esto se resume en $190.378 \mathrm{~kg}$ de fuel oíl que además de presentar como residuo de su combustión $\mathrm{CO}_{2}$, presenta también $\mathrm{SO}_{2}$ (Duda, 1977). El dióxido de azufre puede fijarse en los pulmones agravando síntomas del asma y causando bronquitis en las personas (Connecticut department of energy and enviromental protection, 2013).

Al producir geo-polímero se evita toda la emisión de gases de combustión emitido por la descomposición térmica de los carbonatos, ya que la única fuente de $\mathrm{CO} 2$ es el $0.03 \%$ contenido en la materia orgánica del material (Palomo, Fernandez JImenez, \& Criado, 2004).

Se ha demostrado, que un material con esas características es posible conseguirlo, aplicando caolín ya que este presenta una relación $\mathrm{SiO} 2 / \mathrm{Al} 2 \mathrm{O} 3$ de 1.2 aplicada por varios autores, definiendo con esto a la geopolimerización como cadenas de aluminio-silicato en las cuales el número de moléculas de silicato pueden variar siendo estas: 1 molécula de silicio polisialato, 2 moléculas de silicio polisialato siloxo, 3 moléculas de silicio polisialato disiloxo todas estas con relación a una molécula de aluminio estabilizadas por unión de un metal alcalino (Davidovits, 1994). Estas cadenas se forman por co-polimerización del aluminio y especies de silicato que proceden de la disolución de la alúmina y silicio presente en la constitución química de los materiales en un medio alcalino (P. Soares, 2007)

Considerando que el Ecuador es rico en arcillas, la primera parte del estudio consistiría en encontrar la química del activador aplicando matrices experimentales tipo $2^{\mathrm{k}}$ y mediante análisis mineralógicos buscar una relación $\mathrm{SiO} 2 / \mathrm{Al} 2 \mathrm{O} 3$ de alguna arcilla cerámica similar o superior al caolín para llevar a cabo la polimerización.

\section{Hipótesis}

Partiendo de una arcilla y una solución alcalina se puede generar un material aglomerante con propiedades mecánicas similares o superiores al cemento portland.

\section{Objetivos}

- Obtener la composición y formulación de la solución activadora de la reacción de geo-polimerización.

- Determinar características de materiales para la generación de geo-polímeros.

- Observar comportamiento en pruebas de resistencia.

\section{MATERIALES Y MÉTODOS}

Para analizar la química el activador se emplea un Caolín blanco tamizado en malla ASTM 200, que es un aluminosilicato con un contenido de $\mathrm{SiO} 2$ de $35.49 \%$ y Al2O3 de 33.76\%. Como activador alcalino se utilizó una solución de hidróxido de sodio $(\mathrm{NaOH})$, mientras que se probó con varias vehículos para desestabilizar los enlaces sílice alúmina entre los que tenemos silicato de sodio $\left(\mathrm{Na}_{2} \mathrm{O}_{3} \mathrm{Si}\right)$ y acetato de aluminio (C6H9AlO6) dosificados mediante el diseño experimental tipo $2 \mathrm{k}$. Para probar la solución activadora se procedió a hacer el análisis de una arcilla cerámica disgregada en un molino de martillos 2 veces consecutivas y tamizada en malla ASTM 200 asegurando la uniformidad de la muestra, con un contenido de $\mathrm{SiO} 2$ de $42.17 \%$ y $\mathrm{Al} 2 \mathrm{O} 3$ de $23.6 \%$.

\subsection{DISEÑO DE LA MATRIZ EXPERIMENTAL}

El silicato de sodio y acetato de aluminio fueron disueltos 0.2 moles en soluciones de hidróxido de sodio de 2.5 molar y 5 molar según la estructura de la matriz. Las soluciones se probaron en caolín por su alto contenido en alúmina y por servir como base para estudios a autores como Palomo, La matriz experimental aplicada se muestra en la Tabla 1.

Tabla 1. Matriz experimental variando ion activador

\begin{tabular}{ccccc}
$\mathbf{N}^{\mathbf{0}}$ & $\begin{array}{c}\mathbf{N a}_{2} \mathbf{O}_{\mathbf{3}} \mathbf{S i} \\
(\mathbf{m o l})\end{array}$ & $\begin{array}{c}\mathbf{C 6 H 9 A l O 6} \\
(\mathbf{m o l})\end{array}$ & $\begin{array}{c}\text { Solución de } \\
\mathbf{N a O H} \mathbf{2 . 5} \mathbf{M} \\
(\mathbf{m l})\end{array}$ & $\begin{array}{c}\text { Solución de } \\
\mathbf{N a O H} \mathbf{5 M} \\
(\mathbf{m l})\end{array}$ \\
\hline 1 & 0,2 & 0 & 15 & 0 \\
\hline 2 & 0 & 0,2 & 15 & 0 \\
\hline 3 & 0,2 & 0,1 & 15 & 0 \\
\hline 4 & 0,1 & 0,2 & 15 & 0 \\
\hline 5 & 0,2 & 0 & 0 & 15 \\
\hline 6 & 0 & 0,2 & 0 & 15 \\
\hline 7 & 0,2 & 0,1 & 0 & 15 \\
\hline 8 & 0,1 & 0,2 & 0 & 15 \\
\hline
\end{tabular}

Matriz experimental de 8 experimentos variando en moles Aluminio y silicato y la concentración de la solución de hidróxido de sodio como activadores de reacción. 
Se pesó y homogenizó en seco los componentes de cada experimento, para luego agregar lentamente la solución activadora hasta alcanzar el punto de pasta.

Tabla 2. Preparación de muestras

\begin{tabular}{|ccc|}
\hline $\mathbf{N}^{\mathbf{0}}$ & Caolín (mol) & Solución Activadora \\
\hline 1 & 0,3 & punto plástico \\
\hline 2 & 0,3 & punto plástico \\
\hline 3 & 0,3 & punto plástico \\
\hline 4 & 0,3 & punto plástico \\
\hline 5 & 0,3 & punto plástico \\
\hline 6 & 0,3 & punto plástico \\
\hline 7 & 0,3 & punto plástico \\
\hline 8 & 0,3 & punto plástico \\
\hline
\end{tabular}

Dosificación de las posibles soluciones activadoras sobre el caolín hasta punto plástico

Hallada la solución activadora, se realizó un análisis mineralógico para determinar la actividad de regresión o la reactividad tanto del caolín como de la arcilla cerámica a utilizar, codificada como Arcilla-S.

Tabla 3. Análisis de difractometría de rayos X

\begin{tabular}{cccc} 
Muestra & $\begin{array}{c}\text { Cristalina } \\
(\%)\end{array}$ & $\begin{array}{c}\text { Amorfo } \\
(\mathbf{\%})\end{array}$ & $\begin{array}{c}\text { \% Actividad } \\
\text { por regresión }\end{array}$ \\
\hline Caolín & 43,1 & 56,9 & 80,31 \\
\hline Arcilla-S & 39.2 & 60.8 & 84,74 \\
\hline
\end{tabular}

Muestra el porcentaje de cristalinidad y parte amorfa del caolín y de la Arcilla-S, también el porcentaje de actividad del material obtenido por regresión.

Con esto se puede determinar que ambos materiales tienen una capacidad de reacción similar, por lo tanto se estima que así como se active la reacción geopolimérica en el caolín también lo haga en la Arcilla-S, aplicando el mismo procedimiento.

\subsection{OBSERVACIÓN DE GEO POLIMERIZACIÓN EN FRESCO}

La observación del inicio de geo-polimerización en fresco se realizó en el caolín. Se agregó agua a una muestra de alrededor de $10 \mathrm{~g}$ de caolín de malla 200 hasta punto líquido, luego 2 o $3 \mathrm{ml}$ de solución activadora, observando un cambio de viscosidad en la matriz liquida del caolín.

\subsection{ENSAYO DE RESISTENCIA A COMPRESIÓN}

Para estas pruebas, la concentración de la solución activadora es de $7.5 \% \mathrm{P} / \mathrm{P}$, usando la solución de hidróxido de sodio $2.5 \mathrm{M}$ como medio alcalino. La dosificación de la nueva pasta es de relación $2: 1$, aplicando un tiempo de curado de 3 y 5 horas a $180^{\circ} \mathrm{C}$, reduciendo el tiempo y aumentando la temperatura, variante de $\operatorname{los} 85^{\circ} \mathrm{C}$ y las 20h utilizadas en los experimentos de A. Palomo (2004). Después de este periodo las briquetas fueron retiradas de la estufa y se las dejo enfriar por 20 minutos. Para el ensayo de rotura se utilizó la Prensa para Resistencia a la Compresión de marca ELE y modelo ACCUTEK 250. Según norma ASTM C109, método normalizado de ensayo de resistencia a compresión de morteros de cementos hidráulicos.

\section{RESULTADOS Y DISCUSIONES}

Las pruebas realizadas sobre los geo-polímeros fueron: comportamiento de las briquetas al ser sumergidos en agua por 12 horas, observación del comportamiento de las muestras en fresco al agregar la solución activadora y ruptura de las briquetas por compresión.

\subsection{RESISTENCIA Al AGUA}

Con esta prueba se pudo determinar que la estructura del material es diferente al original, comprobando así un cambio en su estructura molecular. Debido a que si se colocara una muestra únicamente de arcilla en poco tiempo en presencia de agua esta se diluiría en pocas horas. Los resultados se muestran en la Tabla 4.

Tabla 4. Resultados de la matriz experimental

\begin{tabular}{|cccccc}
$\mathbf{N}^{\mathbf{0}}$ & $\begin{array}{c}\mathbf{N a} \mathbf{O}_{3} \mathbf{S i} \\
(\mathbf{m o l})\end{array}$ & $\begin{array}{c}\text { C6H9AlO6 } \\
(\mathbf{m o l})\end{array}$ & $\begin{array}{c}\text { Solución } \\
\mathbf{d e} \\
\mathbf{N a O H} \\
\mathbf{2 . 5} \mathbf{~ M} \\
(\mathbf{m l})\end{array}$ & $\begin{array}{c}\text { Solución } \\
\mathbf{d e} \\
\mathbf{N a O H} \\
\mathbf{5 M}(\mathbf{m l})\end{array}$ & $\begin{array}{c}\text { Resistencia } \\
\text { al agua }\end{array}$ \\
\hline 1 & 0,2 & 0 & 15 & 0 & No \\
\hline 2 & 0.1 & 0,2 & 15 & 0 & No \\
\hline 3 & 0,2 & 0,1 & 15 & 0 & No \\
\hline 4 & 0.2 & 0 & 15 & 0 & Si \\
\hline 5 & 0,2 & 0 & 0 & 15 & No \\
\hline 6 & 0.1 & 0,2 & 0 & 15 & No \\
\hline 7 & 0,2 & 0,1 & 0 & 15 & No \\
\hline 8 & 0.2 & 0 & 0 & 15 & No \\
\hline
\end{tabular}

Muestra los resultados de la matriz experimental al exponer los experimentos en agua 
Las muestras de caolín se mantuvieron siete días a temperatura y humedad ambiente, procediendo a sumergirlas en agua durante 12 horas, en el lapso de 24 horas el experimento número 4 mostró impermeabilidad; que tiene caolín y solución activadora hasta el punto plástico. Los demás experimentos fracasaron, permitiéndonos descartar al ion aluminio en un medio alcalino como activador de polimerización.

Si bien la alcalinidad de la solución activadora es primordial debido a que una leve traza de grupo $-\mathrm{OH}$ hidroliza rápidamente el $\mathrm{SiH}_{4}$ hidruro de sílice, lo cual activa al silicio, teniendo en cuenta la facilidad con que el hidruro de sílice se activa en presencia de grupos $-\mathrm{OH}$ medio alcalino; es de esperar que cuando se encuentra en forma de un silicato alcalino esta sal se haga soluble (Albert \& Geofrey, 1978). La presencia de iones -OH en la disolución del silicato ayuda a ionizar los iones de silicio como también a atacar o disolver el aluminio presente en el caolín, el ion alcalino se encarga de llenar las cargas negativas que deja la copolimerización entre el aluminio y el sílice.

Teóricamente la sílice proveniente del activador es el encargado de activar a los aluminosilicatos presentes en: caolín, arcillas, cenizas y de más materiales que se han utilizado para geo-polimerización en un medio alcalino. Por lo que el experimento número 4 fue replicado e introducido en agua un lapso de 12 días sin presentar ninguna alteración en sus propiedades mecánicas. El agua de inmersión de la probeta presento un alto grado de alcalinidad, capaz de causar dermatitis de contacto en las manos. El experimento permaneció sin alteración sumergido en agua, comprobando la generación de geo-polímero basado en aluminio y sílice. El comportamiento de este experimento es coincidente con el estudio realizado por A. Palomo (2004) donde demuestra que al agregar una solución activadora con una composición de un Álcali y silicato de sodio (waterglass) $15 \%$, se observa en dicha muestra la presencia de microestructuras y aumento de la densidad de las mismas lo que indicaría geo-polimerización.

\subsection{ENSAYO DE RESISTENCIA A COMPRESIÓN}

Se ha mantenido la concentración de álcali en todos los experimentos realizados que es una variante de las pruebas realizadas por A. Palomo (2004) y Ashley Russell (2014).

En las dos arcillas se observan niveles de relación sílice alúmina de 1.15 y 1.78 respectivamente, que comparados con relaciones usadas A. Palomo (2004) y P. Soares (2007) son relativamente bajas .Los resultados obtenidos se pueden observar en la Figura 1.

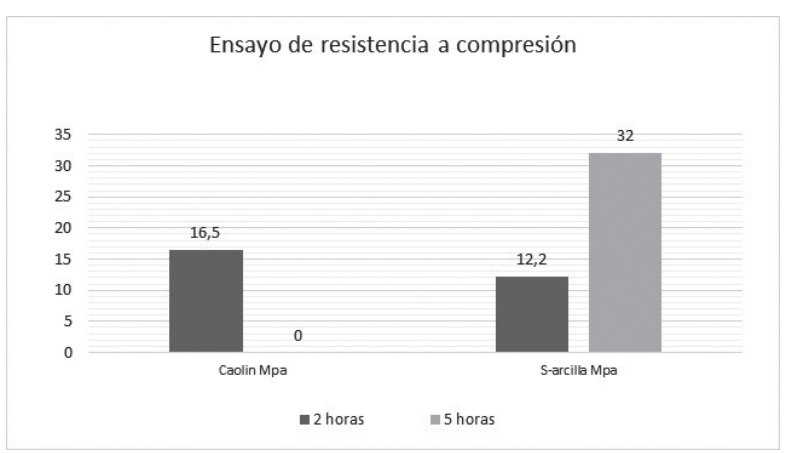

Figura 1. Pruebas de resistencia a la compresión

Los resultados de las pruebas de compresión después de 2 y 5 horas de curado en los dos tipo s de arcillas utilizadas.

Analizando en la Figura 1 la diferencia de resistencia en la prueba de ruptura por compresión se puede observar una mayor resistencia en el caolín a las dos horas (16.5 $\mathrm{MPa})$ que en la S-arcilla (12.2 $\mathrm{MPa})$, esto se puede explicar por la alta reactividad de caolín (80.36) y su parte amorfa (56.9\%), que permite mayor velocidad de geo-polimerización en esta muestra debido a que es más sencillo que se produzca una reacción en un mineral amorfo que un mineral cristalizado.

Analizando los datos de 5 horas de curado se observa que la prueba de compresión de la S-arcilla alcanza una resistencia de $32 \mathrm{MPa}$ superando a la resistencia de los ensayos obtenidos por P. Soares (2007) a los 28 días. Al comparar este resultado con los obtenidos por Ashley Russell (2014) se evidencia una menor resistencia que su experimento de $40 \mathrm{MPa}$ a los siete días. Esto nos sugiere la necesidad de un mayor tiempo de curado para mejores resultados.

La briqueta realizada con caolín después de 5 horas de curado presenta deformaciones, una capa externa accidentada y frágil con un centro duro y muy poco poroso, pero con una forma que imposibilita la prueba de ruptura. Colocando esta muestra en agua se puede observar que la capa externa es soluble, todo lo contrario ocurre con el material debajo de esta capa. Este comportamiento puede deberse a una reacción acelerada y excesiva de geo-polimerización lo cual produciría una red cristalina en la parte externa con un contenido alto de ion alcalino en su formación, razón por la cual dicha capa no presenta resistencia al agua. El material de esta briqueta situado debajo de esta capa, presenta resistencia al agua y se observa menor cantidad de poros que las demás briquetas, 
lo que indica un grado mayor de polimerización (Palomo, Fernandez JImenez, \& Criado, 2004).

Se podría deducir observando la briqueta de caolín que en este proceso de curado la reacción de geo polimerización se produce desde el exterior al interior de la briqueta.

\section{CONCLUSIONES}

El ion silicato en un medio alcalino es el responsable de originar la reacción de geo polimerización en materiales arcillosos con una relación $\mathrm{SiO}_{2} / \mathrm{Al}_{2} \mathrm{O}_{3}$ desde 1.15 o mayor como es el caso de la Arcilla-S. Los resultados evidencian briquetas con propiedades de resistencia mayores son los que tienen menor contenido de alúmina. Alcanzando resistencias mayores de las requeridas para el cemento. Estas briquetas no muestran alteración al ser sumergidas en agua por al menos 10 días.

La reacción de geo-polimerización se acelera con la temperatura pudiendo reducir el tiempo de curado de 20 horas a $85^{\circ} \mathrm{C}$ a 5 horas a $180^{\circ} \mathrm{C}$ con mejores resultados. La reacción de geo-polimerización se produce desde el exterior hacia el interior de la briqueta.

Es necesario estudiar más a fondo el activador debido a la liberación del álcali que se evidencia cuando las briquetas son sumergidas en agua.

De igual manera el estudio de la velocidad de geo polimerización y su avance en las briquetas para determinar el tiempo con el cual se obtenga una reacción completa y se pueda garantizar su máxima resistencia. Aunque la resistencia del material obtenido sea mayor que la del cemento tradicional son necesarios más estudios para que pueda ser utilizado en construcciones

\section{AGRADECIMIENTOS}

Nuestros más sinceros agradecimientos a: Ing. Catalina Peñaherrera, por la ayuda científica prestada durante la realización del proyecto, Ing. Jorge Delgado por la ayuda en la redacción del documento, A Hans Turba por la ayuda prestada en la redacción del abstract, y especialmente al Ing. Patricio Ruiz y al Ing. Esteban Alvear por la ayuda técnica prestada.

\section{REFERENCIAS}

1. Albert, C. F., \& Geofrey, W. (1978). Quimica Inorganica avanzada. Mexico.

2. Ashley Russell Kotwal1), Y. J. (2014). Characterization and Early Age Physical Properties of Ambient Cured. International Journal of Concrete Structures and Materials. doi:DOI 10.1007/s40069014-0085-0

3. Connecticut department of energy and enviromental protection. (13 de Mayo de 2013). www.ct.gov/deep. Recuperado el 24 de abril de 2014, de http://www. ct.gov/deep/lib/deep/air/siprac/2013/fuel_sulfur_ factsheet.pdf

4. Davidovits, J. (1994). Geopolymer Institute. Firts international conference on Alkaline cements and concretes (págs. 2-10). Kiev: Geopolimer institute. Recuperado el 24 de Abril de 2014, de PROPERTIES OF GEOPOLYMER CEMENTS: http://www. geopolymer.org/library/technical-papers/8-alkalinecements-and-concretes-properties-of-geopolymercements

5. Duda, W. (1977). Manual tecnologico del cemento. Barcelona: Romargraf s.a.

6. P. Soares, A. P. (1 de 8 de 2007). Geopolimeros basados en residuos de la produccion de aridos ligeros. Materiales de construccion, 58, 23-34.

7. Palomo, A., Fernandez JImenez, A., \& Criado, M. (8 de Marzo de 2004). "Geopolimero": Una unica base quimica y diferentes estructuras. Mater Construcc, 54(275), 77-92. Obtenido de http://materconstrucc. revistas.csic.es.

Recepción: 8 de Septiembre de 2015

Aceptación: 30 de Octubre de 2015 